\title{
Dynamic walking of multi-humanoid robots using BFGS Quasi-Newton method aided Artificial potential field approach for uneven terrain
}

Abhishek kumar Kashyap ( $\sim$ AKKashyapmech@gmail.com )

National Institute of Technology Rourkela https://orcid.org/0000-0001-8805-0640

Dayal R Parhi

National Institute of Technology Rourkela

\section{Research Article}

Keywords: Hybrid APF-BFGS Quasi-Newton technique, Uneven terrain, Dining philosophers controller, Multi humanoid system, Dynamic obstacle

Posted Date: March 7th, 2022

DOI: https://doi.org/10.21203/rs.3.rs-347037/v1

License: (c) (i) This work is licensed under a Creative Commons Attribution 4.0 International License.

Read Full License 


\title{
Dynamic walking of multi-humanoid robots using BFGS Quasi-Newton method aided Artificial potential field approach for uneven terrain
}

\author{
Abhishek Kumar Kashyap · Dayal R. Parhi
}

\begin{abstract}
The humanoid robot garners paramount interest because of its ability to mimic human-like behavior in realtime environments. In this paper, a hybridized Artificial Potential Field (APF)-Broyden Fletcher Goldfarb Shanno (BFGS) Quasi-Newton technique is being proposed for the trajectory planning of the humanoid robot. The proposed methodology combines the faster local search BFGS QuasiNewton method with the global search APF method for an efficient and faster-hybridized trajectory planning technique. The humanoid robot herein is being tested in a multi-humanoid working space having uneven surfaces. The data obtained from the sensors concerning the location of obstacles and the target are at first imparted to the APF method, which supplies an intermediate turning angle dependent on the predesigned training model of the APF method. The obtained turning angle is then fed into the BFGS quasi-newton method to produce an optimum turning angle, which inevitably guides the humanoid robots to the target by keeping a minimum safe distance from the obstacles. Multi-humanoid robots are employed in an environment having random static obstacles, with unique targets for them to reach. The proposal of using a multihumanoid system arises the chance of inter-collision among the humanoids. To get rid of this situation, the dining philosophers controller is being implemented. The simulations and experiments are carried using the proposed technique to ratify the efficacy of the proposed technique. The experimental and simulation results yield a suitable acceptance rate under $5 \%$. In comparison with the previously used navigational technique, it proves to be comfortably skillful and robust for the trajectory planning of the humanoid robot.
\end{abstract}

Keywords Hybrid APF-BFGS Quasi-Newton technique · Uneven terrain · Dining philosophers controller · Multi humanoid system · Dynamic obstacle

Abhishek Kumar Kashyap

Mechanical Engineering Department, National Institute of Technology, Odisha, 769008, Rourkela, India

Dayal R Parhi

Mechanical Engineering Department, National Institute of Technology, Odisha, 769008, Rourkela, India

\section{Introduction}

Humanoid robots are being extensively used for various applications where human-like behavior is being implemented. Humanoids are provided with an ability to perceive environment conditions via the usage of various external sensors and provide output feedback on the basis of received inputs. Humanoids are used in multiple areas of manufacturing and production lines as well as quality analysis processes for their ability to deliver consistent results in monotonous activities. The implementation of these humanoids thus improves productivity as well as efficiency in various industrial processes. It can be more safely planted in toxic and hazardous environments to perform human-like tasks. In current times, humanoids are being used in multiple areas like production lines, construction sites, underwater tasks, repairing shops, etc. Due to its extensive usage in significant fields, navigational strategy for a humanoid robot is a major concern as it decides the minimum time required by the robot to traverse between two locations while avoiding the obstacles in its path

The navigational strategy employed for the robot is an essential parameter in deciding the time required and the energy spent in traversing a specific path. Various researchers have implemented the use of many AI (Artificial Intelligence) techniques for the trajectory planning process because of its accuracy in delivering results. Trajectory planning has stayed as one of the toughest regions in the research of robots. Simultaneously, in relation to the trajectory planning of humanoids, it turns out to be more complicated as it is entirely dissimilar from the trajectory planning of mobile robots, including complex joint developments as opposed by the wheel development. In the course of the most recent couple of years, a few researchers have endeavored trajectory planning analysis of various types of robots. Trajectory planning techniques are sorted as conventional techniques and artificial intelligence techniques dependent on their strategy in taking care of issues examined. While conventional techniques are acquired from essential measurable strategies, artificial intelligence (AI) techniques are generally a population-based technique. Conventional techniques (Kumar et al., 2018), for example, Artificial potential field (APF), Voronoi diagram (VD), Regression Analysis (RA) (Kumar, Sahu, et al., 2018), etc. are known as to find the result which converges faster. AI techniques, for example, Adaptive neuro-fuzzy inference system (ANFIS) (Pandey et al., 2019), genetic algorithm (GA) (Deepak and Parhi, 2012), particle swarm optimization (PSO) 
(Deepak and Parhi, 2012), Spider monkey optimization approach (SMO) (Lagaza et al., 2020), Wind-driven optimization (WDO) (Bej et al., 2020), etc. are known to take care of issues with more noteworthy exactness in spite of the fact that they may require additional time in merging towards the solution. Various similarly used techniques (Abhishek K Kashyap and Pandey, 2018) are discussed here.

Peng et al. (2019) have used radio frequency identification technology for the indoor navigation of mobile robots. The RFID readers acknowledge the position of the mobile robot and avoid obstacles successfully via a double positioning system for adjusting the navigational parameters in real-time. Garibeh et al. (2019) have proposed the path planning of mobile robots based on the positive and negative forces from the final target and the obstacles in the cluttered environment. The final output provides a given acceleration for the locomotion of the robot. Patle et al. (2018) have used the binary code in the matrix format for static and dynamic environment navigation of the mobile robot. The proposed methodology simplifies the cluttered environment into an array, and final simulation results were obtained by applying the code using MATLAB. Algabri et al. (2015) have worked on the combination of various fuzzy techniques consisting of manually constructed fuzzy (M-fuzzy), Neural-fuzzy, PSOfuzzy and Genetic Algorithm fuzzy controller. The simulation results showed better performance for PSO-fuzzy and Neural fuzzy, but the M-fuzzy technique has bested in the real-time scenario. Masmoudi et al. (2016) have used a hybrid fuzzy logic-PI controller alongside an intelligent transport system for path minimization and obstacle avoidance of the mobile robot in a cluttered environment.

Mohanta and Keshari (2019) have proposed a hybrid fuzzy-probabilistic roadmap method (PRM) for the path planning of mobile robot by using the PRM while moving along straight lines and the fuzzy logic while turning around corners. Pandey et al. (2019) have employed an ANFIS controller for the smooth movement of a mobile robot in a cluttered environment. Various UV and IR sensors are also employed to detect obstacles in the current locomotion path. Patle et al. (2018) have proposed a bio-inspired heuristic firefly algorithm for optimum path generation and obstacle avoidance for a mobile robot. The model was presented as a multivariable correlation function for a complex environment. Pandey et al. (2016) have used a Type-2 fuzzy logic controller for improvising the self-learning and reasoning ability during the path generation and obstacle avoidance of a mobile robot in a cluttered environment. Ko et al. (2017) have proposed a novel neural-network-based controller using a recurrent neural network strategy consisting of a multi-layer perceptron for the smooth functioning of a homecare mobile robot, which shows decent functioning ability in new environments. Omrane et al. (2016) proposed the use of a single fuzzy logic controller for the complete path planning and obstacle avoidance of the mobile robot by employing the usage of IR sensors and optical encoders. Almasri et al. (2015) have proposed a fuzzy logic fusion model consisting of a set of distance cameras and range finder cameras for path planning of the mobile robot in an unknown environment.

The whole body control (Kashyap, Parhi, et al., 2020) has been implemented to acquire a dynamic balance of humanoid robot, and further, 3D LIPM (linear inverted pendulum model) (Kashyap, Pandey, et al., 2020) has been employed to obtain a stabilized gait. Bouhajar et al. (2015) have proposed a PID controller with a predictive strategy for the trajectory planning of humanoid robots. Stereo vision (Fakoor et al., 2016) technique has been preferred by authors to obtain safe navigation in any environment. Sun and Roos (2014) have utilized joint stiffness to achieve an energy-efficient balanced gait. And further extended his work by implementing it in uneven and inclined terrains (Sun and Roos, 2018). Biplab et al. (2018) have hybridized a conventional and a nature-inspired technique to obtain an efficient approach which can converge faster and provide optimum results for trajectory planning for humanoid robots. Kusuma et al. (2019) have preferred A start search method to employ it in humanoid robots. The main objective of the authors is to obtain a safe and balanced walking and rerouting it when necessary to get a collision-free path. Parhi et al. (2018) have preferred multiple humanoid robots to examine the navigation using a hybrid metaheuristic technique. Authors (Gutmann et al., 2005) have emphasized on trajectory planning of humanoid robots in a cluttered environment having stair of different height.

A thorough study of the above-mentioned papers reveals that extensive work has been done for the trajectory planning of mobile robots in various environments, and only a handful number of researches has been done for humanoid robots. The humanoid robot research boasts of only a few research papers in which the work is limited to mostly stability and footstep planning. Some navigational researches are present, but that is also limited to single humanoid robots and flat terrains. The implementation of hybrid techniques in trajectory planning is not very popular. The current research work is based on trajectory planning in uneven surfaces, which increases the complexity of the terrain. Due to the complexity in the path planning of humanoids in multi-humanoid working space, a hybridized APF-BFGS Quasi-Newton method is being proposed for the same. The hybridized combines the local search and faster convergence criteria of the BFGS QuasiNewton method with the accurate analysis of the target and obstacles by the APF method. This hybridized technique is used for optimized path planning for a multi-humanoid system in uneven terrains. The hybridization of the APF and BFGS quasi-newton method provides an optimum turning angle as the final output in two steps. Firstly sensors information is fed to APF, which provides an intermediate turning angle. It is again fed into the BFGS quasi-newton method, which provides the final turning angle as output. This output helps the robot to avoid the obstacles with the minimum safest distance from it. The proposed method seems to perfectly applicable to multiple robots traversing in uneven terrain. The technique appears less effective during the situation of a self-collision. To eliminate this situation, a Dining philosophers controller is being used. The Dining philosophers controller decides the priorities in a dynamic environment and implements a collision-free path. Various research works on the APF, BFGS Quasi-Newton and dining philosophers are presented here.

Mcfetridge and Ibhrahim (1998) have proposed an improvised method of Artificial Potential Field, which determines the priority of each obstacle and its further consequences by introducing a variable dependent on the obstacles' coordinates from the robot's position. Authors (Orozco-Rosas et al., 2019) have proposed a combined membrane computing with a genetic algorithm and artificial potential field for efficient path planning of the mobile robot. 
Weerakoon et al. (2015) have proposed an improvisation over the convention APF method, which avoids the robot being stuck in a local minima zone via velocity vector application. Rimon and Koditschek (1992) have proposed a navigational strategy which employed the usage of a feedback control mechanism to provide torque input data to the mobile robot for a collision free-motion. Rimon et al. (1995) have proposed a new control strategy based on the exact tracking of the gradient lines and avoidance of the local minima zone during locomotion to the target point. Kashyap and Pandey (2020) have proposed an iterative search strategy to avoid deadlock situations during path planning of a mobile robot using the TLBO algorithm. Ibrahim et al. (2014) proposed a hybrid algorithm for a local search method whose directional strategy is a culmination of the BFGS Quasi-Newton method and Conjugate Gradient method for a faster and more efficient search method. Nezhad et al. (2013) proposed a hybrid CPSOBFGS algorithm for the trajectory planning strategy of the mobile robot where the CPSO is implemented in the global search, whereas the BFGS is employed in the neighborhood search regions. Liu and $\mathrm{Li}$ (2007) have proposed a hybrid BFGS-SQP algorithm for mobile robot navigation in which the search direction is finalized by the solution of a consistent quadratic problem whilst covering both local and global search criteria. Liu and Nocedal (1989) provided a comparison between the proposed L-BFGS and the conventional BFGS algorithm with conjugate steps and partitioned Quasi-Newton methods which shows the superiority of the former due to low operating cost and global search criteria. Herescu and Palamidessi (2001) have proposed a randomized algorithm which assigns priority to the events performed in the dining philosophers problem and proves to be reliable for any generalized situation. Lehmann and Rabin (1981) proposed an algorithm based on probabilistic terms for obtaining the successful solution of the dining philosophers problem in a more efficient way.

The implementation of a sole AI technique for the path planning of humanoid robots isn't much efficient and takes a very long time to converge at the final results. Often during navigation in the given environment, the humanoid gets stuck in local minima. Hence, this poses a severe problem in complex situations where often the results are inconclusive. The proposed hybridized technique deals with the issue of local minima with a global search methodology. The proposed search method is employed for multiple humanoid systems for traversing between the start point and target to achieve optimum travel length in optimum time. Trajectory planning for a humanoid robot in uneven terrain is yet distant from the robotization that has been introduced in simulated regions. The self-governance of the humanoid robot based on the conditions of terrains can be achieved by the selection of an efficient and robust technique to make acceptable decisions. Therewith, the implementation of the multihumanoid robot in a single terrain should be operated with extra care, because there may be a chance of inter-collision. For that reason, the present article is concentrated on the design and implementation of a hybrid APF-BFGS quasinewton technique to guide the multiple robots to their respective target both in simulated and experimental terrains. The footstep planning for humanoid robots based on inverse kinematics has been presented to walk over the uneven surface. The majority of the researches on humanoid trajectory planning is restricted to movement control with obtaining target and avoiding collision strategies. Although, the present work evaluates smooth walking control alongside avoiding inter-collision of humanoid robots in uneven terrains.

The APF technique is based on the presence of positive and negative potential fields in the given environment, which, as a result, provides optimum direction for the robot to travel in order to reach the final target. The BFGS Quasi-Newton is based on a negative search gradient method that implements a computational approach for achieving the minima condition. The hybridization of the two proposed techniques is implemented for optimum path length and optimum travel time. The framework of the paper is presented as follows: The mathematical formulation of walking over uneven terrain is discussed in Section 2, navigational approaches are discussed in Section 3, along with their hybridization. Section 4 discusses the evaluation of the efficiency of the proposed technique. Section 5 shows the experiments and simulations carried in this regard. Finally, Section 6 shows the comparison of the proposed technique with other standard method and Section 7 presents the conclusion and the future scope of work.

\section{Mathematical formulation Gait generation for walking over the uneven terrain}

The schematic diagram of the humanoid robot is displayed in Fig. 1 with the specification. The mass of torso, left upper limb, left lower limb, left feet, right feet, right lower limb and right upper limb are denoted by $m_{1}, m_{2}, m_{3}, m_{4}, m_{5}, m_{6}$ and $m_{7}$, respectively. The length of the torso, left upper limb, left lower limb, left feet, right feet, right lower limb and right upper limb are denoted by $s_{1}, s_{2}, s_{3}, s_{4}, s_{5}, s_{6}$ and $s_{7}$, respectively. $\beta_{1}$ is the angle of the trunk with the vertical axis, $\beta_{2}$ and $\beta_{3}$ are the angle of the left leg, $\beta_{6}$ and $\beta_{7}$ are the angle of the right leg, and $\beta_{4}$ and $\beta_{5}$ are the angles of the left foot and right foot, respectively. The representation of the robot while walking in the uneven terrain is portrayed in Fig. 2.

\subsection{Trajectory generation during walking over the uneven surface}

The position of each joint is decided by forward kinematics. And the angle of each joint is determined by inverse kinematics. SSP is acquired to research the generation of trajectory from the lower limb to the hip joint for stepping on the uneven surface.

$$
\beta_{6}=\cos ^{-1}\left[\frac{w_{1}\left(s_{6}+s_{7} \cos \psi_{1}\right)+l_{1}\left(s_{7} \sin \psi_{1}\right)}{\left(s_{6}+s_{7} \cos \psi_{1}\right)^{2}+\left(s_{7} \sin \psi_{1}\right)^{2}}\right]
$$

where

$$
l_{1}=s_{6} \cos \beta_{6}+s_{7} \cos \beta_{7}
$$

$w_{1}=s_{6} \sin \beta_{6}+s_{7} \sin \beta_{7}$

$$
\beta_{7}=\beta_{6}-\psi_{1}=\beta_{6}-\cos ^{-1}\left[\frac{l_{1}^{2}+w_{1}^{2}-s_{6}{ }^{2}-s_{7}{ }^{2}}{2 s_{6} s_{7}}\right]
$$




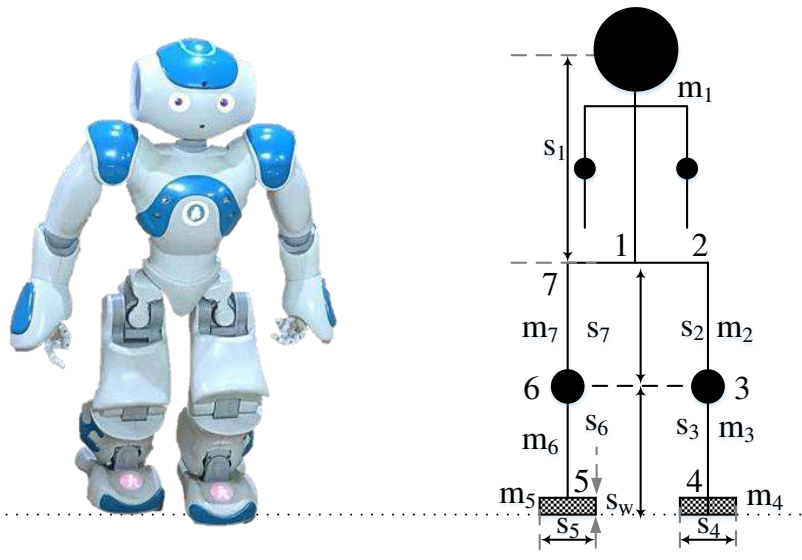

Fig. 1. Schematic representation of humanoid robot NAO with specification

Similarly,

$$
\beta_{3}=\sin ^{-1}\left[\frac{w_{2}\left(s_{3}+s_{2} \cos \psi_{2}\right)+l_{2}\left(s_{2} \sin \psi_{2}\right)}{\left(s_{3}+s_{2} \cos \psi_{2}\right)^{2}+\left(s_{2} \sin \psi_{2}\right)^{2}}\right]
$$

where

$$
l_{2}=s_{2} \cos \beta_{2}+s_{3} \cos \beta_{3}
$$

$w_{2}=s_{2} \sin \beta_{2}+s_{3} \sin \beta_{3}$

$$
\beta_{2}=\beta_{3}-\psi_{2}=\beta_{3}-\cos ^{-1}\left[\frac{l_{2}^{2}+w_{2}^{2}-s_{2}{ }^{2}-s_{3}^{2}}{2 s_{2} s_{3}}\right]
$$

$\beta_{4}=\beta_{5}=0$

ZMP theory is considered to evaluate the dynamic balance of the humanoid robot. ZMP equation for $X$ direction and $Y$ direction has been presented as:

$$
Z M P_{x}=\frac{\sum_{k=1}^{n_{t}} \tau_{k}-\sum_{k=1}^{n_{t}} x_{k} f_{c p_{k} z}^{\perp}-\sum_{k=1}^{n_{t}} z_{k} f_{c p_{k} x}^{\perp}}{\sum_{k=1}^{n_{t}} f_{c p_{k} z}^{\perp}}
$$

$$
Z M P_{y}=\frac{\sum_{k=1}^{n_{t}} \tau_{k}-\sum_{k=1}^{n_{t}} y_{k} f_{c p_{k} z}^{\perp}-\sum_{k=1}^{n_{t}} z_{k} f_{c p_{k} y}^{\perp}}{\sum_{k=1}^{n_{t}} f_{c p_{k} z}^{\perp}}
$$

where $f_{c p_{k} x}^{\perp}=m_{k} \ddot{x}_{k}, f_{c p_{k} y}^{\perp}=m_{k} \ddot{y}_{k}$ and $f_{c p_{k} z}^{\perp}=m_{k}\left(\ddot{z}_{k}-g\right)$, $\left(f_{c p_{k} x}^{\perp}, f_{c p_{k} y}^{\perp}, f_{c p_{k} z}^{\perp}\right)$ is the force acting perpendicular in $(x, y, z)$ direction, $n_{t}$ is the number of links, $g$ is the acceleration due to gravity, $m_{k}$ is the mass of $k^{\text {th }}$ link, and $\ddot{x}_{k}, \ddot{y}_{k}$ and $\ddot{z}_{k}$ is the acceleration of $k^{\text {th }}$ link in $x, y$ and $z$ direction. $\left(x_{k}, y_{k}, z_{k}\right)$ indicates the coordinates of $k^{\text {th }}$ combined mass.

Dynamic balance margin is described as the distance between the point where ZMP acts to the extension of the foot.

$$
\begin{gathered}
D B M_{x}=\left(\frac{s_{4}}{2}-\left|Z M P_{x}\right|\right) \\
D B M_{y}=\left(\frac{s_{w}}{2}-\left|Z M P_{y}\right|\right)
\end{gathered}
$$

The trajectory generation for stepping on the uneven surface has been described. The trajectory for stepping down the uneven surface is similar to the stepping up. The only difference is that while stepping down the uneven surface, the gravity due to acceleration acts opposite to that of motion of the robot. The equation describing the ZMP during stepping down the uneven surface is similar to Eq. 5 and Eq. 6. The value of force in the $z$ direction is different and described as:

$$
f_{c p_{k} z}^{\perp}=m_{k}\left(\ddot{z}_{k}+g\right)
$$

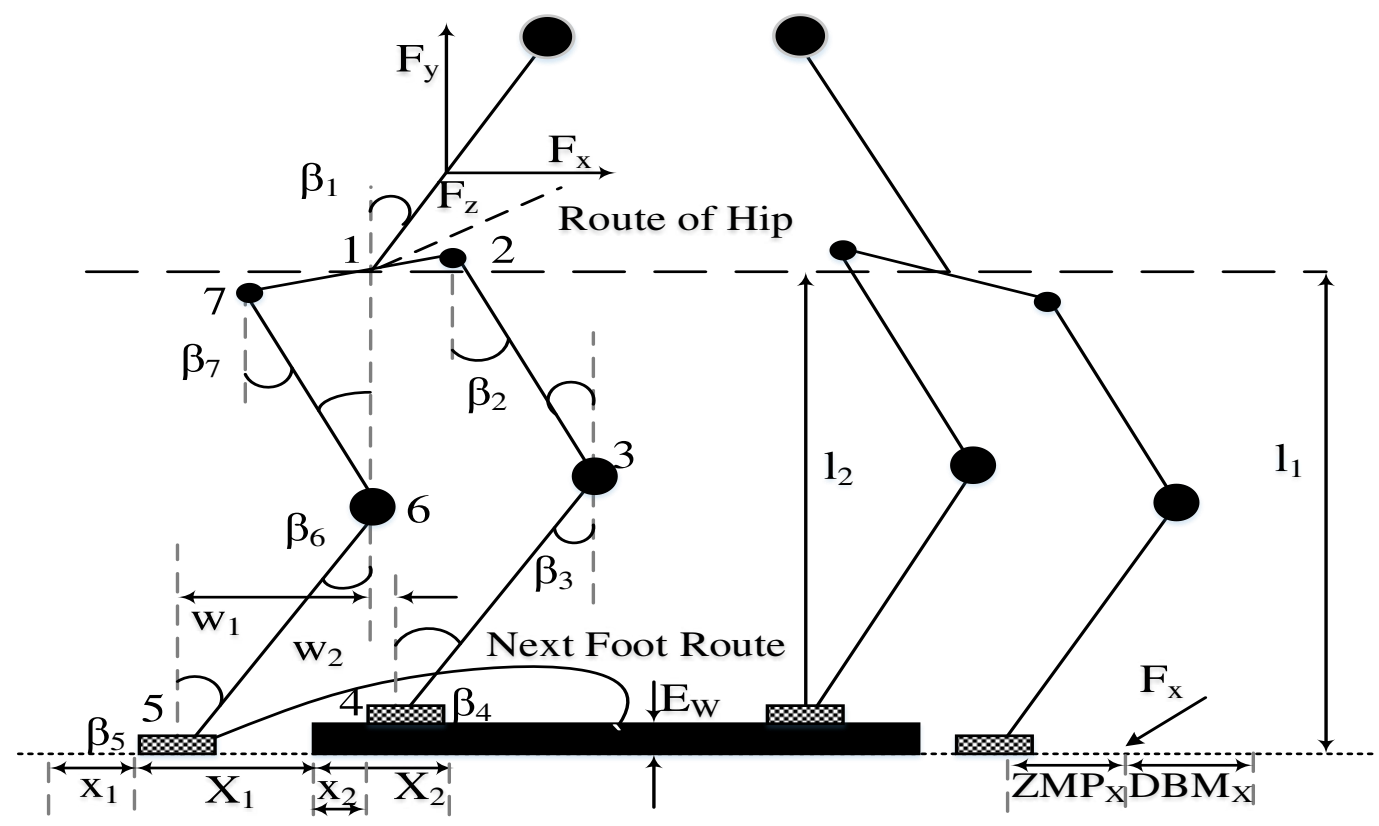

Fig. 2. Schematic diagram of humanoid robot walking over the obstacle 


\section{Proposed approaches for optimization}

A technique utilized for the collision-free trajectory in a cluttered terrain generally has objectives of obtaining target and generating a smooth trajectory to avoid obstacles. Therefore, to fulfill this requirement, the robot should first explore the terrain and reach the target with minimum effort.

\subsection{Artificial Potential Field Method}

The artificial potential field explores the terrain by determining the position of obstacles and targets based on the negative potential field and positive potential field. The distance of obstacles in all directions is fed to the technique, which provides a turning angle as output. Fig. 3 portrays the input and output of the APF method. The APF was first introduced by Khatib in 1986 for controlling the motion of a manipulator arm to avoid collision (Khatib, 1986).

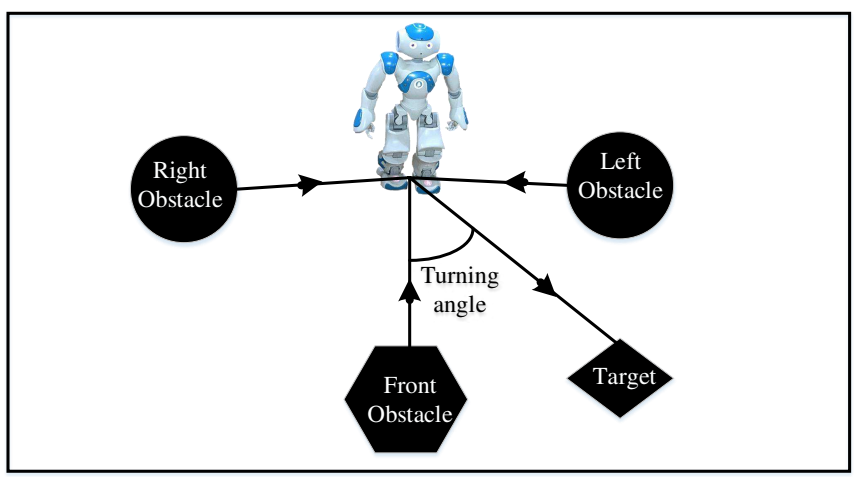

Fig. 3. Inputs and output related to APF method

The classical path planning approach used hereby is the Artificial potential field method. It is based upon the existence of positive and negative forces in the surroundings of the robot. The APF can be used in both static and dynamic environments and can be used for both local and global path planning. The main aim is to derive an energy function for the system in which the robot traverses. The attractive potential function being used here is the quadratic well potential function. The attractive function is defined as:

$$
E_{\text {att }}(x)=\frac{1}{2} k_{1}\left(x-x_{t}\right)^{2}
$$

where, $k_{1}$ is constant, $x_{t}$ is the position vector of the target, $x$ is the position vector of the robot, and $E_{a t t}(x)$ is the energy of the attractive potential field. The attractive force obtained from this gradient formula is:

$$
V_{a t t}(x)=-\nabla E_{a t t}(x)=-k_{1}\left(x-x_{t}\right)
$$

The repulsive potential function is the FIRAS function defined as:

$$
E_{\text {rep }}(x)=\left\{\begin{array}{cl}
\frac{1}{2} k_{2}\left(\frac{1}{d}-\frac{1}{d_{0}}\right)^{2} & \text { if } d \leq d_{0} \\
0 & \text { otherwise }
\end{array}\right.
$$

where, $k_{2}$ is constant, $d_{0}$ is the limit distance of repulsive force influence and $d$ is the shortest distance to the obstacle. The repulsive force thus obtained is:

$$
V_{\text {rep }}(x)=-\nabla E_{\text {rep }}(x)=\left\{\begin{array}{cr}
k_{2}\left(\frac{1}{d}-\frac{1}{d_{0}}\right) \frac{1}{d^{2}} \frac{d(d)}{d x} & \text { if } d \leq d_{0} \\
0 & \text { otherwise }
\end{array}\right.
$$

The extent of the effect of repulsive potential field is much lesser than that of the attractive potential field for easy targeting of goal from a far distance and less interference of negative potential field from affecting the robot's path trajectory when it is at a safe distance from the obstacle. The flowchart of APF method is displayed in Fig. 4.

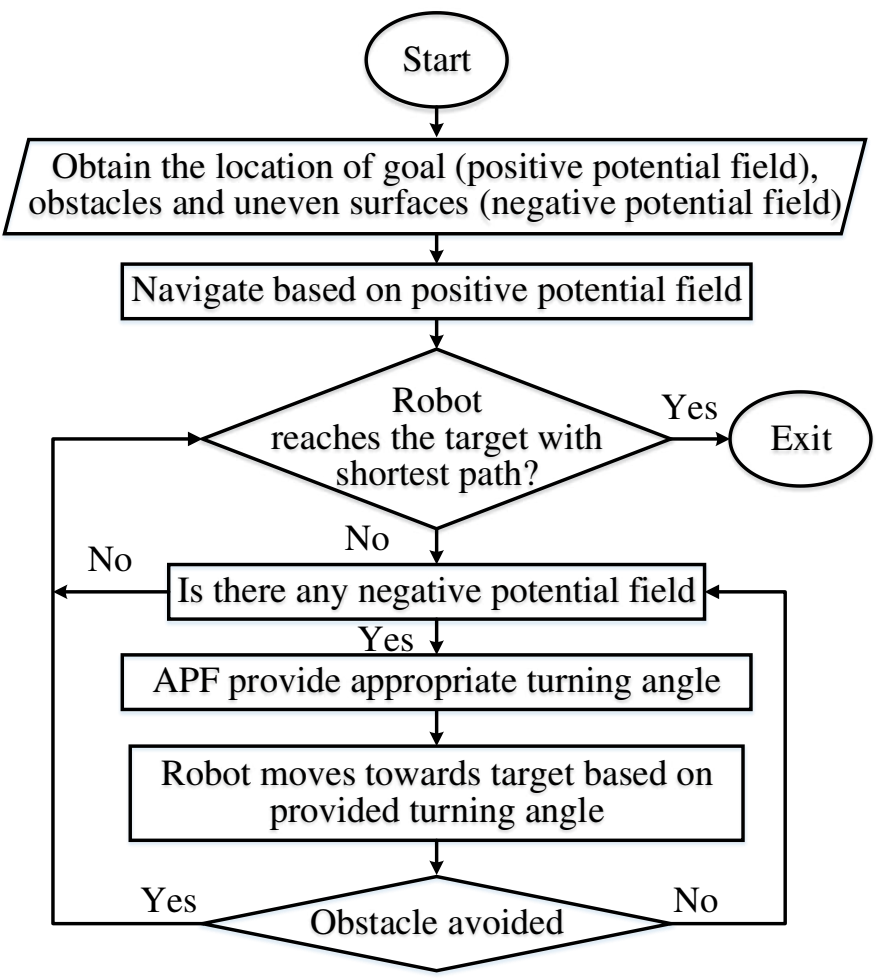

Fig. 4. Flowchart of Artificial potential field method for robot navigation

\subsection{BFGS Quasi-Newton Method}

BFGS quasi newton methods (Shoham et al., 1992) have been obtained through the Newton method. It is a computational iterative technique used for solving nonlinear optimization problems by the usage of the first derivative and Hessian matrix of the objective function. The iteration formula used for the given technique is:

$$
y_{i+1}=y_{i}+s_{i} p_{i}
$$

where, $y_{i}$ is the value at $i^{\text {th }}$ iteration, $y_{i+1}$ is the calculated value at $i+1^{\text {th }}$ iteration, $s_{i}$ is the gait size at $i^{\text {th }}$ iteration and $p_{i}$ is the search direction in the sample space. The search direction given in the method is described as:

$$
p_{i}=-A_{i} d_{i}
$$


where, $A_{i}$ is a positive semi-definite matrix which is inverse to the actual Hessian matrix obtained at the $i^{\text {th }}$ iteration given by $A_{i}=H m_{i}^{-1}$ and $H m_{i}$ represents the Hessian matrix obtained for the objective function. $d_{i}$ is the gradient search direction defined as:

$$
d_{i}=\nabla f\left(y_{i}\right)
$$

The search criterion is described as a product of the negative of positive definite matrix and gradient search direction, ensuring that the search is carried out in the decreasing order given that $\nabla f\left(\mathrm{y}_{i}\right) \neq 0$.

The updation criteria for $H m_{i}$ is selected such that the positive definiteness and the symmetry of the matrix is maintained, it is described as:

$$
\begin{gathered}
H m_{i+1}=H m_{i}+R_{1} \\
I m_{i+1}=I m_{i}+R_{2}
\end{gathered}
$$

where $R_{1}$ and $R_{2}$ are the matrix of rank 1 and rank 2, respectively. $I m_{i+1}$ represents the inverse matrix of the Hessian matrix. The fundamental equation of BFGS quasinewton is portrayed as:

$$
H m_{i+1} \nabla y_{i}=\nabla d_{i}
$$

where $\nabla y_{i}=y_{i+1}-y_{i}$ and $\nabla d_{i}=d_{i+1}-d_{i}$

The hessian matrix has been updated, and the BFGS equation is depicted as:

$$
H m_{i+1}=H m_{i}+\frac{\nabla y_{i} \nabla y_{i}^{T}}{\nabla y_{i}^{T} \nabla d_{i}}-\frac{H m_{i} \nabla d_{i} \nabla d_{i}^{T} H m_{i}}{\nabla d_{i}^{T} H m_{i} \nabla d_{i}}
$$

The BFGS algorithm used here is a local search algorithm in which the probability of finding the exact solution is higher because the modification criteria of $\mathrm{Hm}_{i}$, the matrix take into consideration the recent values of the iteration and follow a linear convergence rate. The algorithm for the BFGS quasinewton method is presented in Fig. 5. The flow chart has been depicted in Fig. 6.

\subsection{Tuning of APF using BFGS-quasi newton method}

The proposed trajectory planning technique proposed in the given study is a culmination of the APF and the BFGS Quasi-Newton method. Trajectory planning is an essential and complex zone in robot analysis and requires the implementation of a proper strategy to obtain desired results within the provided constraints. Generally, implementing a single navigational strategy for path planning results in various problems like slow computation, inaccurate results, etc. To overcome this problem, hybridization of a classical and a computational technique is being done here to improvise the robustness and efficiency of the overall navigation strategy.

The BFGS Quasi-Newton is a computational method that is relatively faster because it searches for minima in the given space according to a given gradient descent formulation. This method, however, depends mostly on the termination criteria provided and is often in the trouble of getting trapped in a local minima condition. The APF technique can be used as a global search method based on the potential fields executed by the target as well as the obstacles on the search path. The presence of an attractive potential field and its applied force on the robot avoid it from being trapped in local minima and reapproach the global minima. The APF technique executes easy predictability and assurance of the final target while making the avoidance of path obstacles easier and specific. The robot initially traverses the path based on the initial search direction obtained from the BFGS Quasi-Newton method. After coming in the vicinity of an obstacle, the APF method provides the robot with a certain turning angle. It redirects its path towards the goal direction as predicted by the presence of the positive potential field. This results in the generation of the optimum travel length as well as the minimum travel time of the robot in the search space whilst achieving accurate results. The tuning mechanism has been depicted in Fig. 7.

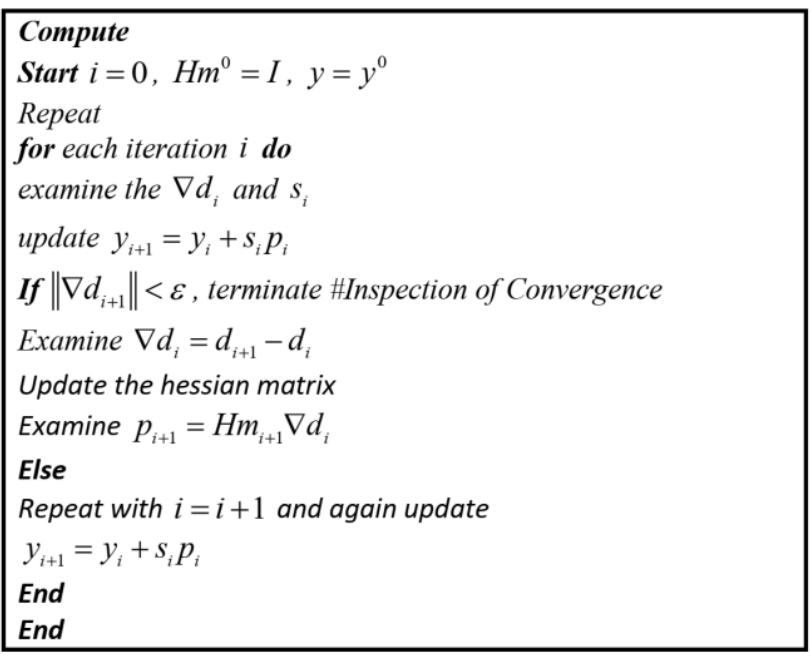

Fig. 5. Algorithm for BFGS quasi newton method

The steps for the working of the hybrid APF-BFGS QuasiNewton controller are listed below:

1 Initialize the start and target position of the humanoid robot.

2 Call the APF algorithm, which determines the target position and provides an optimum directional vector for the robot to follow.

3 The APF detects the presence of any obstacle in the path and calls the BFGS algorithm henceforth.

4 The BFGS algorithm searches the local neighborhood and provides an optimum turning angle for clearing out of the obstacle zone.

5 Once out of the obstacle zone again, the APF is activated, and steps 2-4 are repeated until the target position is reached.

\subsection{Formulation of objective function for safe navigation}

The primary objective of navigation is to reach the target by avoiding collision with the obstacles. In order to obtain safe navigation, the strategy for obstacle avoidance and attaining the target. In the APF technique, the robot should reach a certain distance to the obstacle using a repulsive potential field, which would be reliable. The robot should have the global best position to the nearby obstacles; it is formulated as: 


$$
E_{O A}(i)=\sqrt{\left(x_{O}(i)-x_{R}(i)\right)^{2}+\left(y_{O}(i)-y_{R}(i)\right)^{2}}
$$

where $E_{O A}(i)$ is the objective function for the avoidance of the $i^{\text {th }}$ obstacle by the humanoid robot at $i^{\text {th }}$ position. $\left(x_{O}(i), y_{O}(i)\right)$ is the coordinate of $i^{\text {th }}$ obstacle and $\left(x_{R}(i), y_{R}(i)\right)$ is the coordinate of $i^{\text {th }}$ humanoid robot.
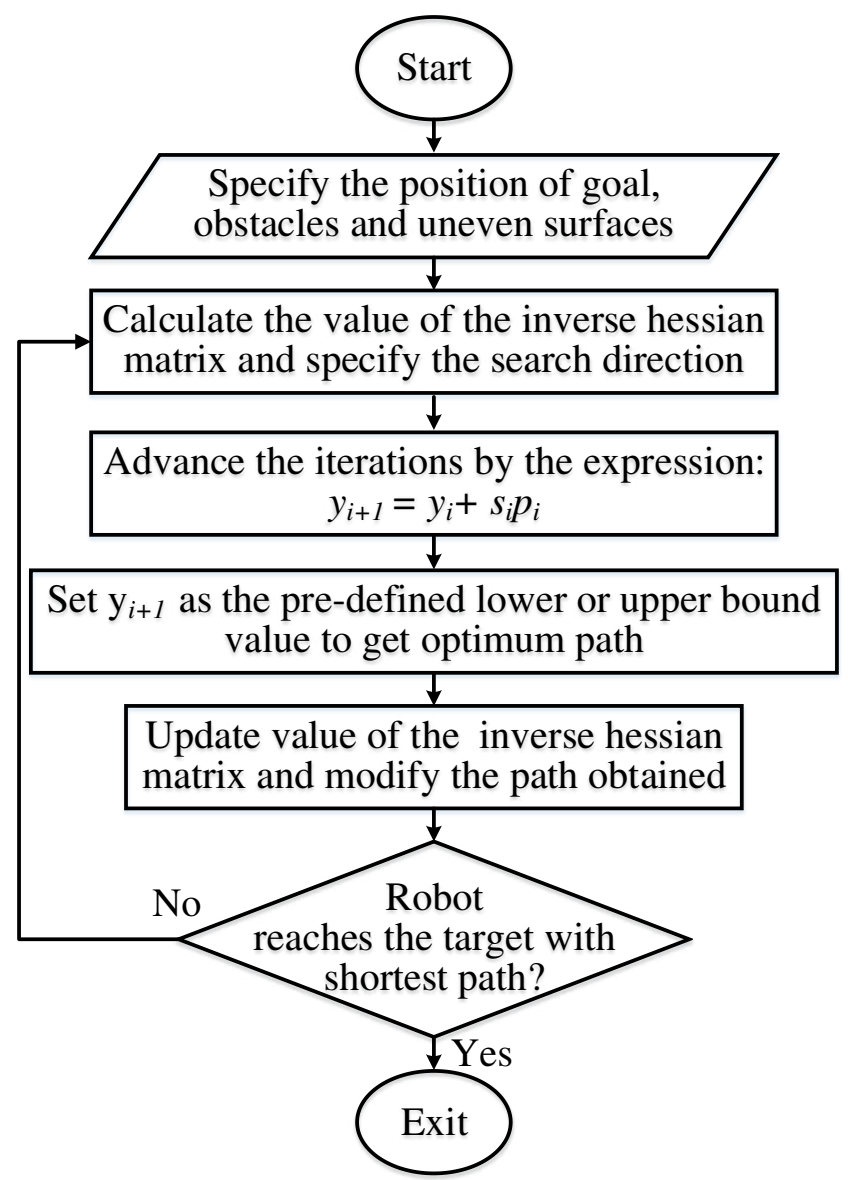

Fig. 6. Flowchart of BFGS quasi newton method for robot navigation

The second most important objective is to reach the target with minimum travel length. This is obtained using an attractive potential field in APF with global optimum value. The global best position of the robot to reach the target with minimum travel length is expressed as:

$$
E_{A T}(i)=\sqrt{\left(x_{T a r}-x_{O}(i)\right)^{2}+\left(y_{T a r}-y_{O R}(i)\right)^{2}}
$$

where $E_{A T}(i)$ is the objective function for attaining target by the humanoid robot at $i^{\text {th }}$ position. $\left(x_{T a r}(i), y_{T a r}(i)\right)$ is the coordinate of the target.

Since both obstacle avoidance and attaining target are essential for the navigation of the humanoid robot. Therefore, the final objective function consists of both behaviors and is represented as:

$$
E_{O F}=w_{1} \sum_{1}^{n} \frac{1}{\operatorname{Min}\left(E_{O A}(i)\right)}+w_{2}\left(E_{A T}(i)\right)
$$

where $w_{1}$ and $w_{2}$ are the repulsive potential field parameter and attractive potential field parameter, respectively, $\operatorname{Min}\left(E_{O A}(i)\right)$ is the minimum possible distance to avoid obstacle and $n$ is the number of obstacles. When the distance between obstacle and robot is more, it will decrease the objective function, and also it will decrease when the distance between the robot and target is less. Therefore, the objective function discussed here is the minimization problem.

\subsection{Dining philosophers controller for avoidance of self- collision}

The dining philosophers problem was first proposed by (Dijkstra, 1971) as a student exam exercise. The problem presents a scenario of 3 or more silent philosophers sitting on a round table with a bowl of spaghetti. A fork is placed between each pair of adjacent philosophers, and the supply of food is assumed to be infinite. The rules imply that food can only be eaten with two forks, and at a time, only one person can use a single fork. For the functioning of this technique, semaphores are introduced whose values vary as per the relationship between the philosopher and the forks being held by $t$. In order to prevent a deadlock condition, a priority basis is being applied to the philosophers while picking up the forks.

The given diagram shows the three philosophers P1, P2, P3 placed on around table with forks F1, F2 and F3 placed between them. Food is placed at the center of the table, which is accessible to each philosopher.

For a multi-humanoid system, the dining philosopher's strategy controller is being used to avoid inter-collisions between the robots when they are present on a common platform. When multiple robots traverse on the same platform, they act as dynamic obstacles to each other, which can't be resolved by using simple obstacle avoidance strategy since the problem of priority allocation arises in this case.

Phases of working of a dining philosophy strategy controller are:

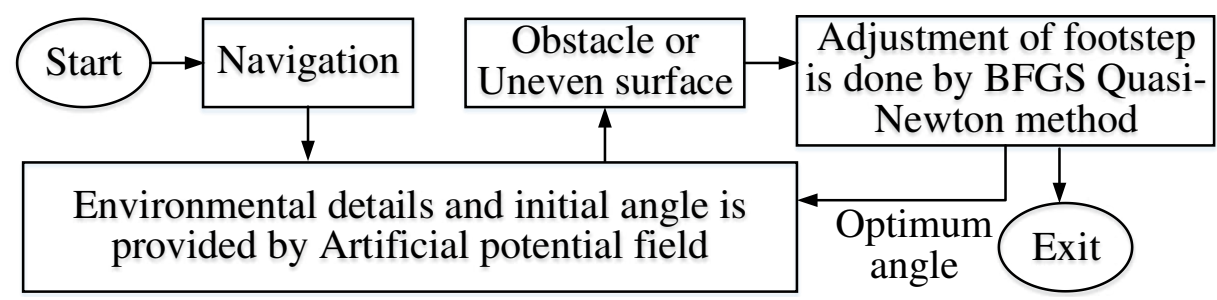

Fig. 7. Mechanism of tuning of APF method using BFGS quasi newton method 


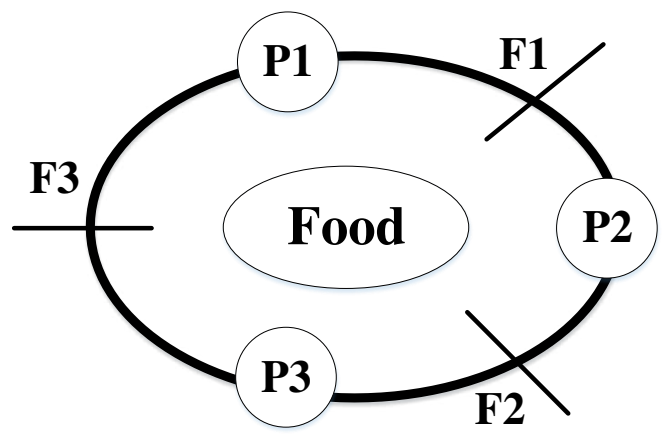

Fig. 8. Fundamental sketch of dining philospher

Phase 1. Robots are at their initial position waiting for the initialization signal.

In this phase, the robots are waiting at their respective start points for the signal to start.

The robots do not know about the presence of another robot. Both simply locate their individual goals and obstacles in their paths.

Phase 2. Robots are traversing to their respective targets in the defined path trajectory as predicted by the proposed technique.

The navigation starts and robots head towards their target by avoiding the obstacles. The robots face static obstacles and take sufficient turns to prevent themselves.

Phase 3. As the robots approach closer to each other, at the proximity limit, the system comes to a deadlock situation.

The adverse situation arises when the robot detects each other. One robot treats as a dynamic obstacle to others. Therefore, one robot stops at its position, and another will move forward.

Phase 4. The proposed controller then negotiates between the given robots according to the criteria given in the algorithm and allots priority to one of the robots.

At this phase, one robot needs to prioritize so that hassle-free navigation can be achieved. Robots stop at the deadlock situation and configure which robot will move first. The robot has a more convenient path to get prioritized and allowed to move first.

Phase 5. The preferred robot continues along its natural path trajectory alongside checking any further obstacles in its path and reaches its target.

Phase 6. The non-preferred robot waits at its position until the preferred robot clears out of its conflict zone, after which it repeats step 5.

In case a third robot comes into the conflict after the two robots are already in a deadlock situation, it is given the lowest priority. The working of the dining philosopher is depicted in Fig. 9.

\section{Evaluation of the efficiency of control techniques}

The hybrid technique has been proposed using the mechanism described in the above section. It provides optimum turning angle and path length to reach the target, which is an essential requirement for trajectory planning. The developed technique needs to be examined to ensure that it will perform efficiently and accomplishes the elements of the task. A computational cost is an essential tool, which should be controlled in order to make the technique effective. It can be controlled using the methods that require less iteration to solve the problem and provide proper stability while taking any turn and crossing over the uneven surface.

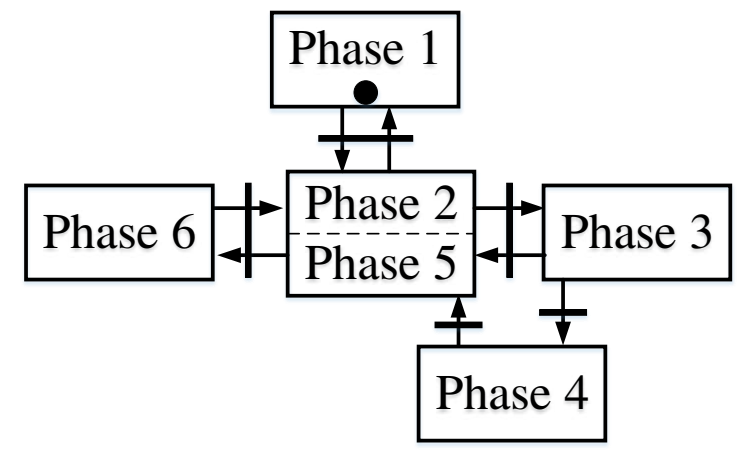

Fig. 9. Schematic working of the dining philosopher's strategy controller

To evaluate the efficiency and robustness of the proposed technique, a terrain consists of start point, target and an obstacle is engaged. The start point is referred to as point $\mathrm{A}$ $\left(X_{a}, Y_{a}\right)$, target as $\mathrm{C}\left(X_{c}, Y_{c}\right)$ and the intermediate point as $\mathrm{B}$, as shown in Fig. 10. The obstacle is presented by a square, and the robot sensing range around the obstacle is shown using a circular ring. The robot starts from point $\mathrm{A}$ and reaches point B using Euclidian distance. The control technique should work to take a turn and reach the target. When a singly developed APF technique works, it takes a longer turn and reaches the target C. But, when the control technique is tuned with BFGS quasi-newton method, the robot comes closer to the obstacle (safest distance) and takes a small turn to reach the target. This small turning angle is optimum, which guides the robot to the target with the shortest possible travel length. It also decreases the computational time and, subsequently, computational cost.

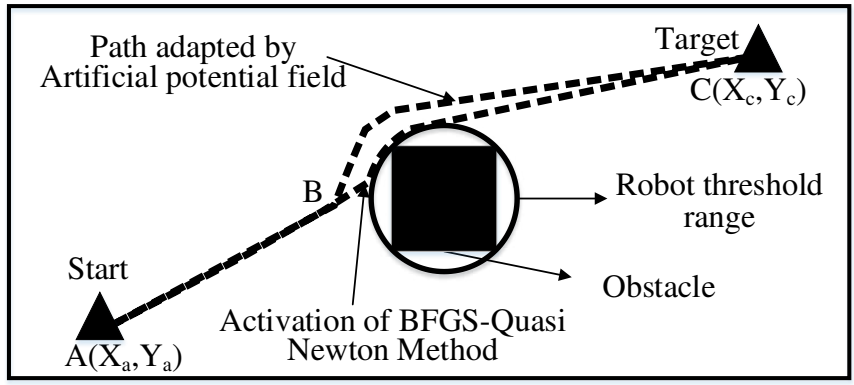

Fig. 10. Implementation of proposed method in robot simulation

The experiment provides an idea that the requirement of footstep placement is necessary during interaction with the obstacle and uneven surface. Because robots cannot make any trajectory to cross it, there would be the possibility to collide with the obstacle or fall. During these conditions, the ZMP should be closer to the ideal ZMP to execute the tasks. The APF technique does not solve the problem of local minima, which 
increases the computational costs and time to complete the tasks. This flaw has been eliminated by tuning it with BFGS quasi-newton method. It helps to converse the result faster and provide better stability to the robot. This hybridization helps in getting optimum path length with a lesser computational cost. The APF technique flawlessly observes the environment and obtains the position of obstacles/ uneven surfaces and the target using negative potential field and positive potential field, respectively. The BFGS quasi-newton method has the advantage that it does not get trapped in local minima, which results in providing the global minima solutions efficiently. The gradient decent vector is utilized to update hessian; it assists the robot in obtaining the next step based on the previous step. When the path accommodates any obstacle or uneven surface, the hessian matrix gets updated frequently. This process helps the robot to select the next foot to cross the obstacles and uneven surface with maximum stability.

The design of footstep placement should be feasible in the matter of leg length, joint speed, joint limits and self-collision. Concerning it, the position of the next step should be designed on the basis of the previous step. The Cartesian maximal joint speed furnishes the result; it is expressed as:

$$
M_{c}\left[\begin{array}{l}
X_{s}-x_{s}(t) \\
Y_{s}-y_{s}(t)
\end{array}\right] \leq\left(t_{c g}-t\right) s_{\max M}
$$

where the Cartesian matrix is denoted by $M_{c} \cdot\left(X_{s}, Y_{s}\right)$, $\left(x_{s}(t), \mathrm{y}_{s}(t)\right)$ and $s_{\max M}$ are referred to as the position of seceding footstep, current footstep, and maximal speed of Cartesian, respectively.

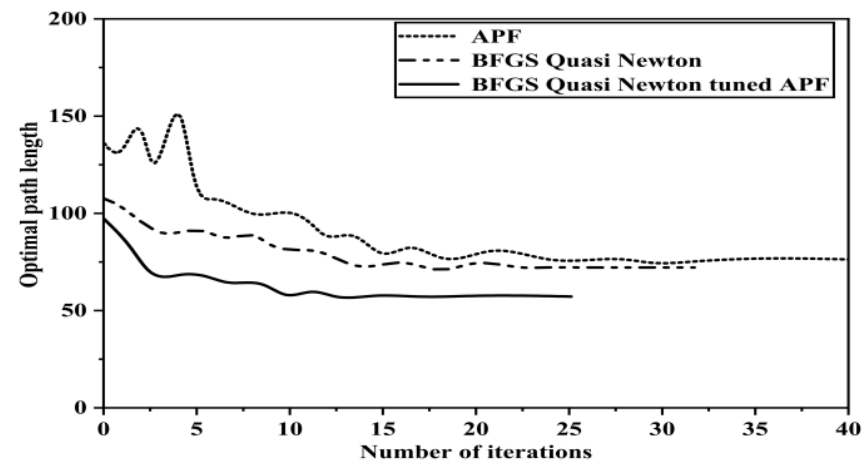

Fig. 11. Convergence curve for APF, BFGS quasi newton and proposed technique

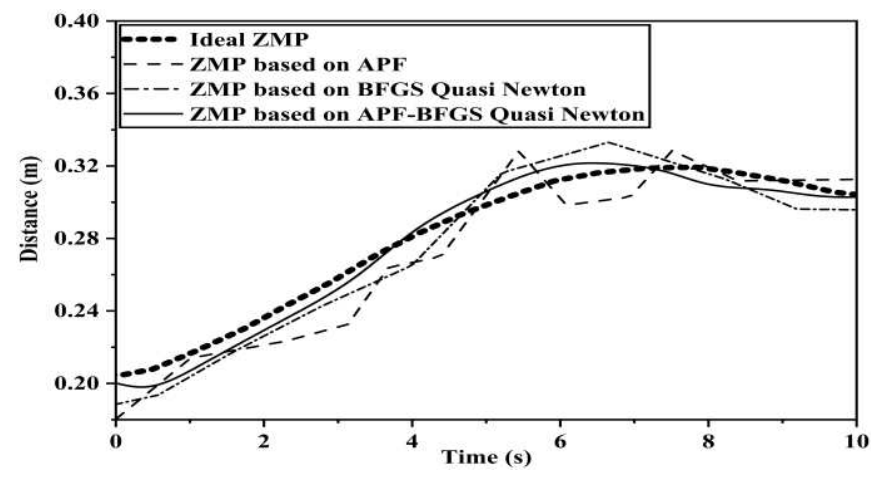

Fig. 12. Comparison of ZMP curve for APF, BFGS quasi newton and proposed technique

The time at which the foot comes in contact with the ground is denoted by $t_{c g}$. The robot navigation using the tuned APF is depicted in Fig. 10. The activity of avoiding the obstacles and passing through the uneven surface is formed by various control methods. The convergence curve to obtain the optimal path length and curve, showing the ZMP variation with time, is depicted in Fig. 11, and Fig. 12. Based on these results, it is concluded the proposed technique converges faster when crossing the obstacles and uneven surfaces. The stabilization achieved by the humanoid robot is compared using ZMP curves. It has been observed that the proposed technique provides better control and, subsequently, stabilization than the singly used method.

\section{Implementation of the proposed hybrid technique}

The robotic platform considered for the purpose of simulation and real-time experiments is the humanoid NAO. The hybrid APF-BFGS quasi-newton method, combined with the Dining philosophers controller, has been effectively modeled. The hybrid model comes into action in case of the presence of obstacles in the vicinity of the humanoid robot. The Dining philosophers controller is activated only when the conflicting situation between robot arises. The following proposed technique has been successfully tested and examined in both simulations and real-time environment.

a. Trajectory planning of multiple humanoid robot in uneven terrains

To validate the efficiency of the proposed hybrid technique, simulations and experiments are being performed on multirobot systems consisting 2 robots in uneven terrains. The conditions of the research in the simulation is taken similar to those in the real-time environment. The humanoids marked N1 and N2 start from their respective positions S1 and S2 and proceeded towards their target $\mathrm{T} 1$ and $\mathrm{T} 2$, respectively. The 3D simulator preferred in this research is WEBOT ("Webots Aldebaran 2.1.4.13 documentation," n.d.). It consists of the NAO model, which completely replicates the real NAO, both in terms of appearance and functions. All motions are similar and completely mimic the behavior of real NAO. Although the proposed hybrid technique is sufficient in clearing the static obstacles and reaching the target, it couldn't perform as predicted in the case of a multi-humanoid system. For this purpose, a Dining philosophers controller is being used, which comes into effect whenever there is a situation of assigning priority among humanoid robots. Simulation results explicit that the robots reach their respective targets safely. The environment model has been created, and static obstacles are set up in simulated terrain, as shown in Fig. 13. In this environment, the robots act as dynamic obstacles to each other, thus activating the Dining Philosophers controller whenever they come in the vicinity of each other.

Thus, the hybridized APF-BFGS quasi-newton algorithm, along with the Dining Philosophers controller, is encoded in the humanoid NAO. The simulation has been carried out, and snapshots of simulation in scene 1 are displayed in Fig. 13. The simulation result is endorsed through a real-time experiment, as shown in Fig. 14. The terrain for the real-time experiment has been designed similarly as simulated terrain such as the position of robots, obstacles, start points and targets. 


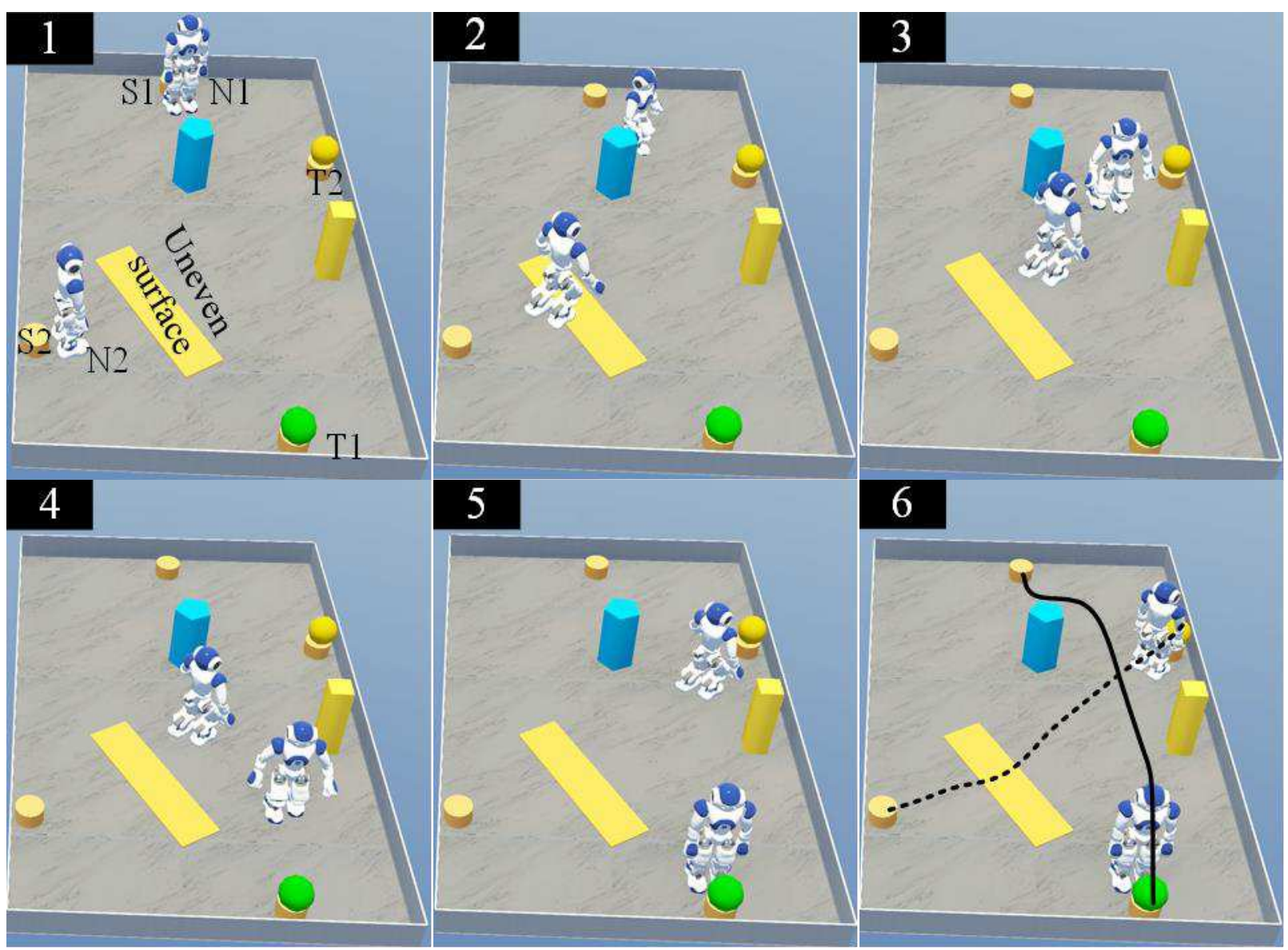

Fig. 13. Snapshot of simulation result of multi-humanoid robot in scene 1

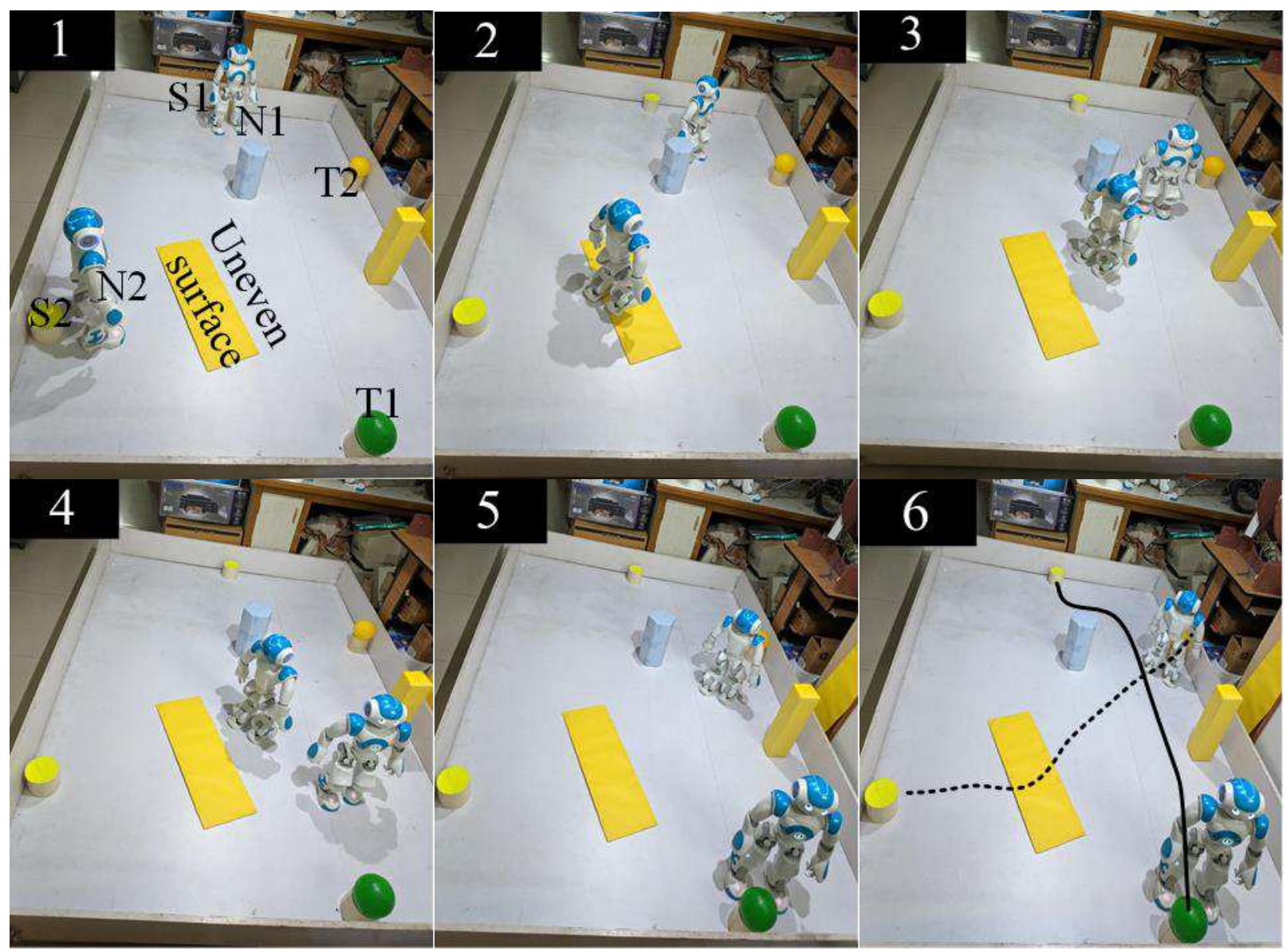

Fig. 14. Snapshot of experimental result of multi-humanoid robot in scene 1 


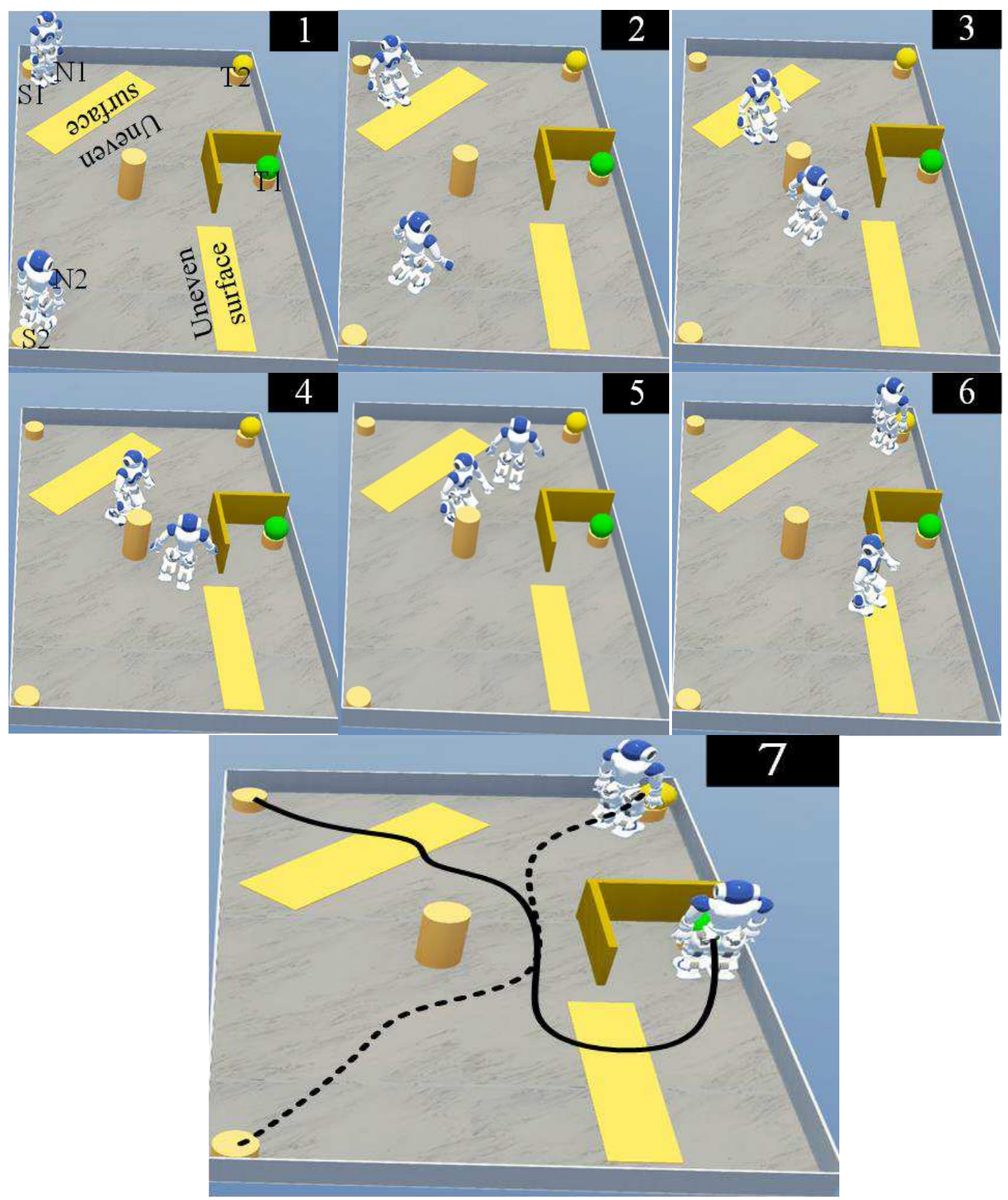

Fig. 15. Snapshot of simulation result of multi-humanoid robot in scene 2

Table 1

Deviation in travel length $(\mathrm{cm})$ between simulation and experimental result in two robots in scene 1

\begin{tabular}{ccccccc}
\hline Sl. & \multicolumn{2}{c}{ Simulation } & \multicolumn{2}{c}{ Experiment } & \multicolumn{2}{c}{ Deviation (\%) } \\
\cline { 2 - 7 } No. & N1 & N2 & N1 & N2 & N1 & N2 \\
\hline 1. & 264 & 257.5 & 271.1 & 265.8 & 2.69 & 3.22 \\
2. & 254.8 & 248.3 & 267.1 & 256.7 & 4.83 & 3.38 \\
3. & 253.4 & 246.7 & 265 & 257.1 & 4.58 & 4.22 \\
4. & 260.2 & 253.7 & 268.4 & 260.9 & 3.15 & 2.84 \\
5. & 258.6 & 252 & 269.8 & 263.6 & 4.33 & 4.6 \\
6. & 253.8 & 247.3 & 266 & 257.1 & 4.81 & 3.96 \\
7. & 266.7 & 260.2 & 276.8 & 271.4 & 3.79 & 4.3 \\
8. & 264.4 & 247.9 & 274.2 & 258.3 & 3.71 & 4.2 \\
9. & 271.1 & 264.6 & 278.1 & 274.7 & 2.58 & 3.82 \\
10. & 255.2 & 250.8 & 264.2 & 260.1 & 3.53 & 3.71 \\
\hline
\end{tabular}

\section{Table 2}

Deviation in travel time (s) between simulation and experimental result in two robots in scene 1

\begin{tabular}{ccccccc}
\hline Sl. & \multicolumn{2}{c}{ Simulation } & \multicolumn{2}{c}{ Experiment } & \multicolumn{2}{c}{ Deviation (\%) } \\
\cline { 2 - 7 } No. & N1 & N2 & N1 & N2 & N1 & N2 \\
\hline 1. & 50.1 & 72.08 & 52.31 & 75.61 & 4.41 & 4.9 \\
2. & 43.27 & 73.27 & 45.19 & 76.49 & 4.44 & 4.39 \\
3. & 41.45 & 71.45 & 43.51 & 73.2 & 4.97 & 2.45 \\
4. & 48.07 & 70.06 & 49.13 & 73.09 & 2.21 & 4.32 \\
5. & 47.15 & 71.18 & 49.09 & 73.1 & 4.11 & 2.7 \\
6. & 41.82 & 71.82 & 43.88 & 74.33 & 4.93 & 3.49 \\
7. & 52.48 & 72.49 & 54.22 & 75.92 & 3.32 & 4.73 \\
8. & 51.6 & 72.58 & 53.7 & 76.05 & 4.07 & 4.78 \\
9. & 54.7 & 70.72 & 56.55 & 73.78 & 3.38 & 4.33 \\
10. & 44.18 & 73.65 & 46.12 & 76.35 & 4.39 & 3.67 \\
\hline
\end{tabular}




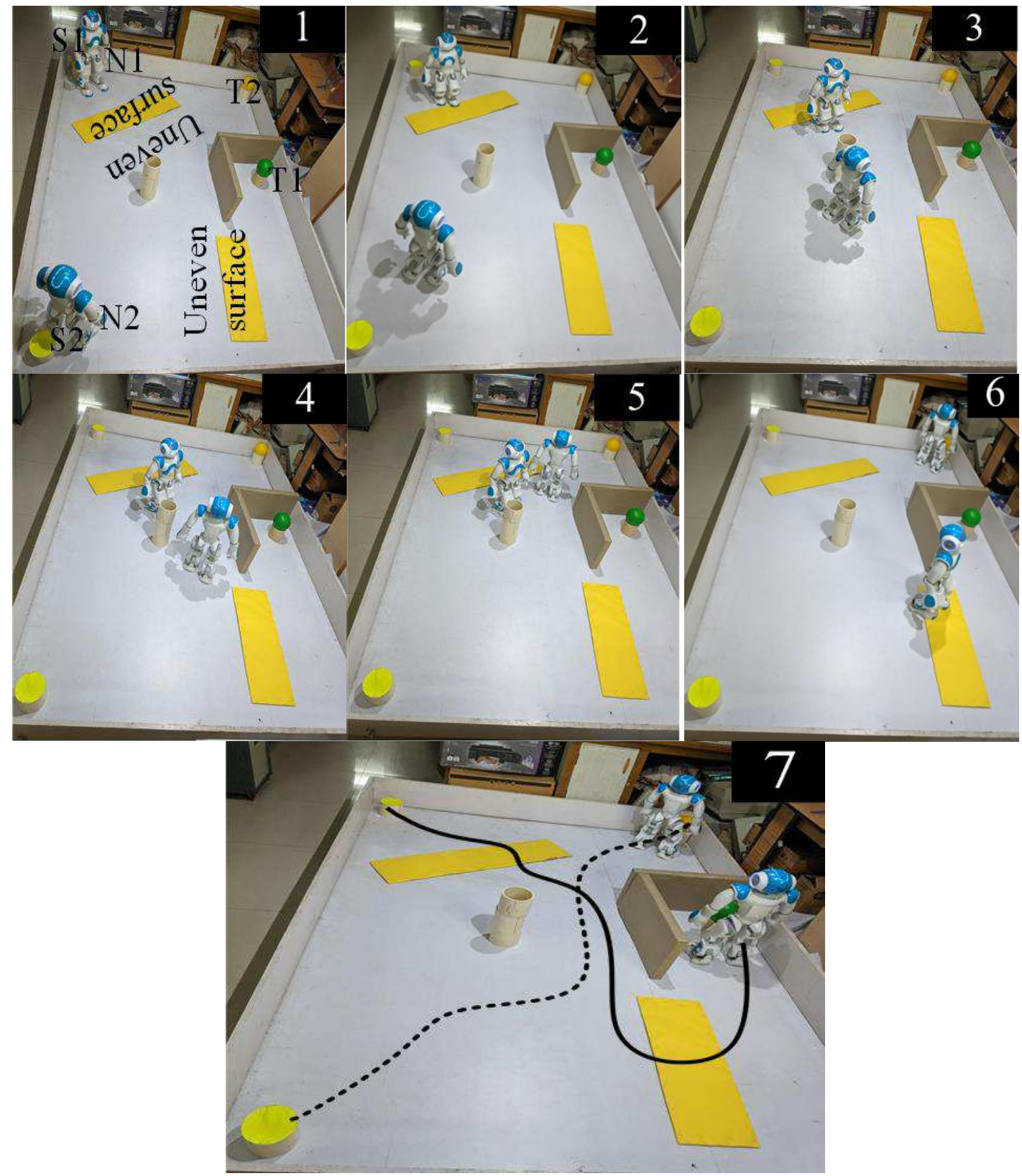

Fig. 16. Snapshot of experimental result of multi-humanoid robot in scene 2

Table 3

Deviation in travel length $(\mathrm{cm})$ between simulation and experimental result in two robots in scene 2

\begin{tabular}{ccccccc}
\hline Sl. & \multicolumn{2}{c}{ Simulation } & \multicolumn{2}{c}{ Experiment } & \multicolumn{2}{c}{ Deviation (\%) } \\
\cline { 2 - 7 } No. & N1 & N2 & N1 & N2 & N1 & N2 \\
\hline 1. & 299.1 & 293.6 & 309.1 & 303.5 & 3.34 & 3.37 \\
2. & 317.8 & 312.3 & 329.5 & 323.9 & 3.68 & 3.71 \\
3. & 308.5 & 293.4 & 319.1 & 307.85 & 3.44 & 4.93 \\
4. & 321.2 & 305.8 & 332.9 & 316.7 & 3.64 & 3.56 \\
5. & 307.4 & 310.2 & 320.3 & 320 & 4.2 & 3.16 \\
6. & 298.9 & 303 & 313.2 & 313.5 & 4.78 & 3.47 \\
7. & 311.3 & 301.9 & 322.3 & 314.7 & 3.53 & 4.24 \\
8. & 315.7 & 315.7 & 325.6 & 327.3 & 3.14 & 3.67 \\
9. & 303.1 & 297.6 & 317 & 311.4 & 4.59 & 4.64 \\
10. & 300.3 & 294.8 & 309.2 & 303.6 & 2.96 & 2.99 \\
\hline
\end{tabular}

\section{Table 4}

Deviation in travel time (s) between simulation and experimental result in two robots in scene 2

\begin{tabular}{ccccccc}
\hline Sl. & \multicolumn{2}{c}{ Simulation } & \multicolumn{2}{c}{ Experiment } & \multicolumn{2}{c}{ Deviation (\%) } \\
\cline { 2 - 7 } No. & N1 & N2 & N1 & N2 & N1 & N2 \\
\hline 1. & 80.1 & 77.2 & 83.18 & 80.28 & 3.85 & 3.99 \\
2. & 87.4 & 83.9 & 89.71 & 86.81 & 2.64 & 3.47 \\
3. & 83.5 & 74.7 & 86.92 & 78.15 & 4.1 & 4.62 \\
4. & 92.6 & 81.9 & 94.7 & 85.02 & 2.27 & 3.81 \\
5. & 82.1 & 83.8 & 85.7 & 86.43 & 4.38 & 3.14 \\
6. & 78.7 & 80.6 & 81.05 & 84.02 & 2.99 & 4.24 \\
7. & 85.2 & 80.1 & 87.92 & 82.8 & 3.19 & 3.37 \\
8. & 86.7 & 88.7 & 89.33 & 91.8 & 3.03 & 3.49 \\
9. & 80.9 & 78 & 83.67 & 80.77 & 3.42 & 3.55 \\
10. & 80.2 & 77.3 & 83.19 & 80.29 & 3.73 & 3.87 \\
\hline
\end{tabular}


Wi-Fi connection has been established to control the NAO, and the controller has been installed using Python language. The navigational data for the simulated terrain is directly recorded from the WEBOT interface, and using a stopwatch and measuring tape for the real-time experiment. The navigational data obtained from both the simulation and experimental cases show the recorded path length and time taken by each robot for further analysis and comparison. The state of conflict arises in Fig. 13 (3) and Fig. 14 (3), where the Dining Philosophers controller is activated, and priority is provided to N1 robot due to its lesser path length among the two robots. The recorded values of travel length and travel time for both the robots are recorded in tabular form for both simulations and experimental cases, as depicted in Table 1 and Table 2, respectively. The obtained values show an average deviation of $5 \%$ in the given test cases, which is acceptable and hence, explains the robustness and accuracy of the proposed technique.

The proposed technique has been examined, and the results as acceptable in terms of trajectory planning in uneven terrain. The proposed technique is further explored in the more complicated terrain consisting of more uneven surfaces and obstacles in scene 2. The presence of these foreign things further increases the complicacy of the terrain. The robot is again examined in simulated terrain using WEBOT software and validated in the real-time experiment. The snapshots of simulation and experiment are depicted in Fig. 15 and Fig. 16. The parameters are recorded in a similar manner in scene 1, and represented in the tabular form in Table 3 and Table 4, showing travel lengths and travel times. The deviation between the simulation and experimental results are recorded under $5 \%$, which shows a good agreement between them. The deviation between them is recorded because of the presence of sound, friction, different parameter recording techniques and $\mathrm{Wi}-\mathrm{Fi}$ connectivity in real-time experiments that is missing in the simulation program.

b. Comparative study of energy efficiency between proposed technique and default controller of NAO

The energy efficiency of the proposed technique is necessarily compared against the default controller of NAO. This comparison will determine whether the proposed controller should be selected. If the consumption of energy by the default controller would be less than the proposed technique, then the selection would not be valid. Therefore, in this section, a comparison of energy consumption for both the controller has been performed for 10 runs, as shown in Fig. 17. In each run, the energy consumption for sagittal and lateral movement has been recorded and displayed in Table 5. The overall improvement of energy defends the selection of the proposed technique. The pitching and rolling movement of the robot consumes different energy, therefore both movements have been considered in this comparison. Overall improvement of about $6 \%$ has been recorded using the proposed technique to validate that the proposed technique is energetically efficient.

\section{Comparison of trajectory adaption with existing technique}

The proposed hybrid technique, along with the Dining philosophers controller, is encoded in the humanoid robot NAO. The obtained results show the effectiveness and accuracy of the proposed technique. For further analysis, the obtained results are compared with those of the established navigational strategies. The paper used in reference to comparison is the Probability and Fuzzy Logic (PFL) trajectory planning technique by Patle et al. (2019). The environment conditions are replicated precisely for the comparison, and simulations are performed on it, as shown in Fig. 18. The terrain consists of 2 robots (R1 and R2) having different goals (Goal 1 and Goal 2).

The final results obtained for the reference paper shows that it successfully clears the obstacles and reaches the final target. However, the comparison of recorded data, as shown in Table 6 , shows the proposed technique taking an edge over the previously developed technique by treading the path via lesser travel length and travel time. The proposed technique takes displays an improvement in travel length and travel time to reach the target as compared to the established technique.

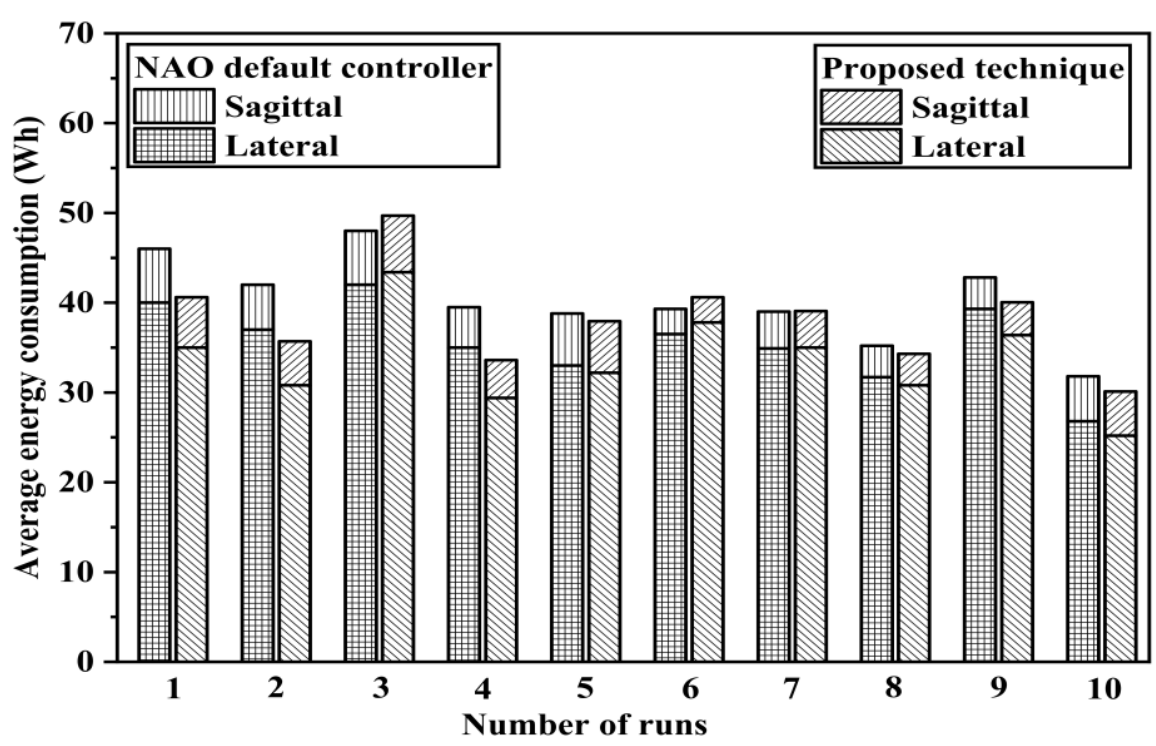

Fig. 17. Comparison of energy consumption between NAO default controller and proposed controller 
Table 5

Comparison of energy consumption (Wh) in sagittal and lateral plane using proposed technique and default controller in NAO humanoid robot

\begin{tabular}{ccccccc}
\hline \multirow{2}{*}{$\begin{array}{c}\text { No. of } \\
\text { Runs }\end{array}$} & \multicolumn{2}{c}{ Default controller } & \multicolumn{2}{c}{ Proposed technique } & \multicolumn{2}{c}{ Improvement (\%) } \\
\cline { 2 - 7 } Lateral & Sagital & Lateral & Sagital & Lateral & Sagital \\
\hline $\mathbf{1 .}$ & 40 & 45.7 & 35 & 40.5 & 12.5 & 11.38 \\
$\mathbf{2 .}$ & 37.2 & 42.1 & 30.8 & 35.2 & 17.2 & 16.39 \\
$\mathbf{3 .}$ & 42.1 & 47.6 & 43.9 & 50 & -4.28 & -5.04 \\
$\mathbf{4 .}$ & 35 & 39.4 & 29.8 & 33.1 & 14.86 & 15.99 \\
$\mathbf{5 .}$ & 32.8 & 38 & 32.5 & 37.6 & 0.91 & 1.05 \\
$\mathbf{6 .}$ & 36.8 & 39.3 & 37.8 & 40.8 & -2.72 & -3.82 \\
$\mathbf{7 .}$ & 35 & 39.1 & 35 & 39.1 & 0 & 0 \\
$\mathbf{8 .}$ & 31.8 & 35.2 & 31 & 34.8 & 2.52 & 1.14 \\
$\mathbf{9 .}$ & 39.6 & 42.5 & 36 & 40 & 9.09 & 5.88 \\
$\mathbf{1 0 .}$ & 27 & 32 & 25 & 30 & 7.41 & 6.25 \\
\hline & & Average & & & $\mathbf{5 . 7 4 9}$ & $\mathbf{4 . 9 2 2}$ \\
\hline
\end{tabular}

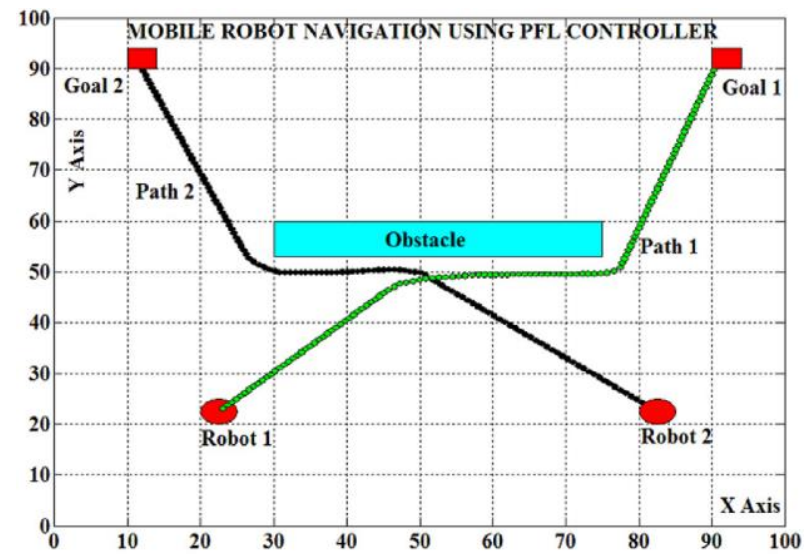

(a) Path selected by Probability and Fuzzy Logic controller (B.K. Patle et al., 2019)

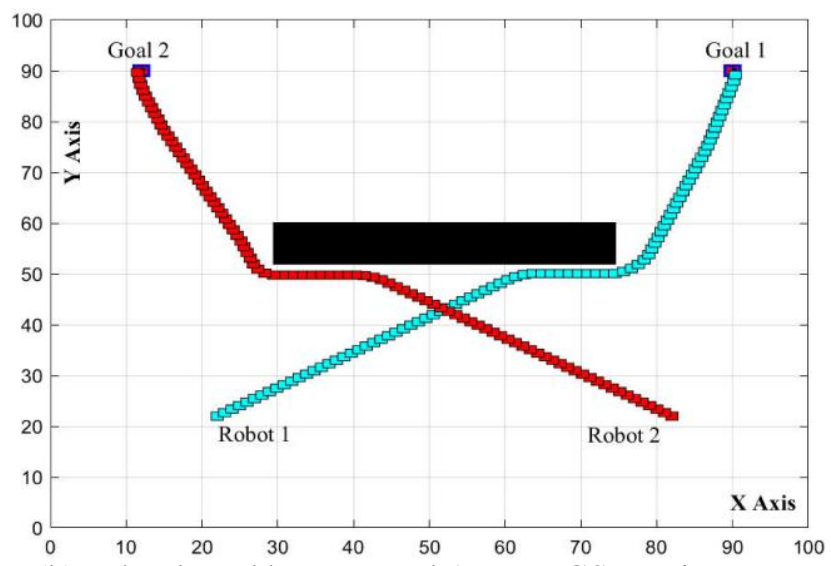

(b)Path selected by proposed APF-BFGS quasi newton technique

Fig. 18. Comparison of path selection between the proposed technique and PFL controller (B.K. Patle et al., 2019)

Table 5

Comparison of travel length and travel time for the proposed technique and PFL controller (B.K. Patle et al., 2019)

\begin{tabular}{|c|c|c|c|c|c|c|c|c|c|c|c|c|}
\hline \multicolumn{5}{|c|}{ SI. PFL controller (B.K. Patle et al., 2019) } & \multicolumn{4}{|c|}{ Proposed technique } & \multicolumn{4}{|c|}{ Improvement (\%) } \\
\hline \multirow[t]{2}{*}{ No. } & \multicolumn{2}{|c|}{ Travel length } & \multicolumn{2}{|c|}{ Travel time } & \multicolumn{2}{|c|}{ Travel length } & \multicolumn{2}{|c|}{ Travel time } & \multicolumn{2}{|c|}{ Travel length } & \multicolumn{2}{|c|}{ Travel time } \\
\hline & R1 & $\mathbf{R 2}$ & R1 & R2 & R1 & $\mathbf{R 2}$ & R1 & $\mathbf{R 2}$ & R1 & $\mathbf{R 2}$ & R1 & $\mathbf{R 2}$ \\
\hline 1. & 171.5 & 186.7 & 27.64 & 30.06 & 160.1 & 170.3 & 26.18 & 28.81 & 6.65 & 8.78 & 5.28 & 4.16 \\
\hline 2. & 171.5 & 186.7 & 27.64 & 30.06 & 172.3 & 187.4 & 28.33 & 30.19 & -0.47 & -0.37 & -2.5 & -0.43 \\
\hline 3. & 171.5 & 186.7 & 27.64 & 30.06 & 158.8 & 174.1 & 25.19 & 28.12 & 7.41 & 6.75 & 8.86 & 6.45 \\
\hline 4. & 171.5 & 186.7 & 27.64 & 30.06 & 151.6 & 172.4 & 23.45 & 26.65 & 11.6 & 7.66 & 15.16 & 11.34 \\
\hline & & & & Average & & & & & 6.297 & 5.705 & 6.7 & 5.38 \\
\hline
\end{tabular}

The lesser travel time required in the proposed technique reduces the computational time of the problem, which ultimately reduces the computational cost and hence proves to be economical. Since uneven terrain was considered for the comparison, it proves that the hybrid APF-BFGS quasinewton algorithm is more efficient and economical for any environment condition.

\section{Conclusion}

1. Trajectory planning has become an essential research field for nearly all robot researchers to ease human struggle in completing tiresome and complicated jobs.
2. The hybrid APF-BFGS quasi-newton technique is successfully developed, applied and tested for multihumanoid robots system on uneven terrains with satisfactory results.

3. The proposed technique provides an optimum turning angle using 2 steps. Initially, APF provides an intermediate turning angle by taking sensory data as input, which is further fed to the BFGS quasi-newton method. It provides the final turning angle, which helps the robot to refrain from obstacles.

4. Dining philosopher controller has been combined with the hybrid technique, activates in a situation of conflict, 
and assigns priority to one of the robots to prevent intercollision amongst them.

5. A total of 500 iterations have been carried out for training the hybrid algorithm to find the optimum turning angle to avoid an obstacle.

6. The robustness and accuracy of the proposed technique are being concluded on several parameters like obstacle avoidance, target-directed angle, turning angle, etc.

7. The simulations and experiments are carried out in two different terrains consisting of random static obstacles and uneven surfaces. Results obtained via hybrid technique show an acceptable deviation of under $5 \%$.

8. The proposed technique has been compared against the default controller of NAO based on energy. The results show the supremacy of $6 \%$ in sagittal and lateral movement.

9. The comparison of the proposed hybrid technique with the established navigational technique shows the superiority of the proposed methodology in parameters of travel length and travel time.

10. It can be inferred that the proposed navigational strategy is more efficient for the trajectory planning of a multihumanoid robot system on uneven terrains.

11. In the future, more techniques can be hybridized to get better results by implementing them in a cluttered environment with inclined terrains.

\section{Compliance with ethical standard}

Conflict of interest: The authors declare that they have no conflict of interest.

Ethical approval: This article does not contain any studies with human participants or animals performed by any of the authors.

Informed consent: Informed consent was obtained from all individual participants included in the study.

\section{Authorship contributions}

Abhishek Kumar Kashyap: Conceptualization, Methodology, Software, Writing- Original draft preparation, Visualization, Investigation, Software, Validation.

Dayal R. Parhi: Supervision, Writing- Reviewing and Editing

\section{References}

Algabri M, Mathkour H, Ramdane H and Alsulaiman M (2015) Comparative study of soft computing techniques for mobile robot navigation in an unknown environment. Computers in Human Behavior 50: 4256.

Almasri M, Elleithy K and Alajlan A (2015) Sensor fusion based model for collision free mobile robot navigation. Sensors (Switzerland) 16(1).

Bej N, Pandey A, Kashyap AK, Parhi DR, N Bej APAKKDRP, Bej N, ... Parhi DR (2020) Optimum Navigation of Four-Wheeled Ground Robot in Stationary and Non-stationary Environments Using Wind-Driven Optimization Algorithm. In Innovative Product Design and Intelligent Manufacturing Systems (pp. 931-941). Springer.
Bouhajar S, Maherzi E, Khraief N, Besbes M and Belghith S (2015) Trajectory Generation using Predictive PID Control for Stable Walking Humanoid Robot. Procedia Computer Science 73(Awict): 86-93.

Deepak B and Parhi D (2012) PSO based path planner of an autonomous mobile robot. Open Computer Science 2(2): 114-134.

Dijkstra EW (1971) Hierarchical ordering of sequential processes. Acta Informatica 1(2): 115-138.

Fakoor M, Kosari A, Jafarzadeh M, Pandey A, Parhi DR, Das PK, ... Pugazhenthi S (2016) Walking Navigation System of Humanoid Robot using Stereo Vision based Floor Recognition and Path Planning with MultiLayered Body Image. Proceedings - IEEE International Conference on Robotics and Automation 6(1): 592-597.

Garibeh MH (2019) A Potential Field Simulation Study for Mobile Robot Path Planning in Dynamic Environments. In 2019 20th International Conference on Research and Education in Mechatronics (REM) (Vol. 5, pp. 1-8). IEEE.

Gutmann JS, Fukuchi M and Fujita M (2005) Real-time path planning for humanoid robot navigation. IJCAI International Joint Conference on Artificial Intelligence 1232-1237.

Herescu OM and Palamidessi C (2001) On the generalized dining philosophers problem. In Proceedings of the twentieth annual ACM symposium on Principles of distributed computing - PODC '01 (pp. 81-89). New York, New York, USA: ACM Press.

Ibrahim MAH, Mamat M and Leong WJ (2014) The hybrid BFGS-CG method in solving unconstrained optimization problems. Abstract and Applied Analysis 2014.

Kashyap Abhishek K and Pandey A (2018) Different NatureInspired Techniques Applied for Motion Planning of Wheeled Robot: A Critical Review. International Journal of Advanced Robotics and Automation 3(2): 110.

Kashyap Abhishek K and Pandey A (2020) Optimized Path Planning for Three-Wheeled Autonomous Robot Using Teaching-Learning-Based Optimization Technique. In Advances in Materials and Manufacturing Engineering (pp. 49-57).

Kashyap Abhishek Kumar, Pandey A, Chhotray A and Parhi DR (2020) Controlled Gait Planning of Humanoid Robot NAO Based on 3D-LIPM Model. SSRN Electronic Journal.

Kashyap Abhishek Kumar, Parhi DR and Kumar S (2020) Dynamic Stabilization of NAO Humanoid Robot Based on Whole-Body Control with Simulated Annealing. International Journal of Humanoid Robotics 17(03): 2050014.

Khatib O (1986) The potential field approach and operational space formulation in robot control. In Adaptive and Learning Systems (pp. 367-377). Springer.

Ko B, Choi HJ, Hong C, Kim JH, Kwon OC and Yoo CD (2017) Neural network-based autonomous navigation for a homecare mobile robot. 2017 IEEE International Conference on Big Data and Smart Computing, BigComp 2017 403-406.

Kumar A, Kumar PB and Parhi DR (2018) Intelligent Navigation of Humanoids in Cluttered Environments 
Using Regression Analysis and Genetic Algorithm. Arabian Journal for Science and Engineering 43(12): 7655-7678.

Kumar PB, Parhi DR, Sethy M, Chhotray A, Kant Pandey K and Sahu C (2018) Humanoid Navigation: An Intelligent Computer Vision Based Approach. IEECON 2018 - 6th International Electrical Engineering Congress 1-4.

Kumar PB, Sahu C and Parhi DR (2018) A hybridized regression-adaptive ant colony optimization approach for navigation of humanoids in a cluttered environment. Applied Soft Computing 68: 565-585.

Kusuma M, Riyanto and Machbub C (2019) Humanoid Robot Path Planning and Rerouting Using A-Star Search Algorithm. In Proceedings - 2019 IEEE International Conference on Signals and Systems, ICSigSys 2019 (pp. 110-115). IEEE.

Lagaza KP, Kashyap AK and Pandey A (2020) Spider Monkey Optimization Algorithm Based Collision-Free Navigation and Path Optimization for a Mobile Robot in the Static Environment. In Advances in Mechanical Engineering (pp. 1459-1473).

Lehmann D and Rabin MO (1981) On the advantages of free choice: A symmetric and fully distributed solution to the dining philosophers problem. Conference Record of the Annual ACM Symposium on Principles of Programming Languages 133-138.

Liu DC and Nocedal J (1989) On the limited memory BFGS method for large scale optimization. Mathematical Programming, Series B 45(3): 503-528.

Liu T and Li D (2007) Convergence of the BFGS-SQP method for degenerate problems. Numerical Functional Analysis and Optimization 28(7-8): 927944.

Masmoudi MS, Krichen N, Masmoudi M and Derbel N (2016) Fuzzy logic controllers design for omnidirectional mobile robot navigation. Applied Soft Computing 49: 901-919.

McFetridge L and Yousef Ibrahim M (1998) New technique of mobile robot navigation using a hybrid adaptive fuzzy-potential field approach. Computers and Industrial Engineering 35(3-4): 471-474.

Mohammad Nezhad A, Aliakbari Shandiz R and Eshraghniaye Jahromi A (2013) A particle swarmBFGS algorithm for nonlinear programming problems. Computers and Operations Research 40(4): 963-972.

Mohanta JC and Keshari A (2019) A knowledge based fuzzy-probabilistic roadmap method for mobile robot navigation. Applied Soft Computing 79: 391-409.

Omrane H, Masmoudi MS and Masmoudi M (2016) Fuzzy Logic Based Control for Autonomous Mobile Robot Navigation. Computational Intelligence and Neuroscience 2016: 1-10.

Orozco-Rosas U, Montiel O and Sepúlveda R (2019) Mobile robot path planning using membrane evolutionary artificial potential field. Applied Soft Computing 77: 236-251.
Pandey A, Kashyap AK, Parhi DR and Patle BK (2019) Autonomous mobile robot navigation between static and dynamic obstacles using multiple ANFIS architecture. World Journal of Engineering 16(2): 275286.

Pandey A, Kumar S, Pandey KK and Parhi DR (2016) Mobile robot navigation in unknown static environments using ANFIS controller. Perspectives in Science 8: 421-423.

Parhi DR, Sahu C and Kumar PB (2018) Navigation of multiple humanoid robots using hybrid adaptive swarmadaptive ant colony optimisation technique. Computer Animation and Virtual Worlds 29(2): 1-20.

Patle BK, Parhi DRK, Jagadeesh A and Kashyap SK (2018) Matrix-Binary Codes based Genetic Algorithm for path planning of mobile robot. Computers and Electrical Engineering 67: 708-728.

Patle BK, Parhi DRK, Jagadeesh A and Kashyap SK (2019) Application of probability to enhance the performance of fuzzy based mobile robot navigation. Applied Soft Computing 75: 265-283.

Patle BKK, Pandey A, Jagadeesh A and Parhi DRR (2018) Path planning in uncertain environment by using firefly algorithm. Defence Technology 14(6): 691-701.

Peng J, Qin Y, Wei Q, He Q, Wan Z and Jiang H (2019) RFID-based indoor mobile robot navigation. International Journal of RF Technologies 10(1-2): 1-8

Rimon E and Koditschek DE (1992) Exact Robot Navigation using Artificial Potential Functions. IEEE Transactions on Robotics and Automation 8(5): 501-518.

Rimon E, Koditschek DE, Guldner J, Utkin VI, McFetridge L, Yousef Ibrahim M, ... Nassiraei AAF (1995) Sliding Mode Control for Gradient Tracking and Robot Navigation Using Artificial Potential Fields. IEEE Transactions on Robotics and Automation 8(3): 501518.

Shoham M, Li CJ, Hacham Y, Kreindler E and Weill R (1992) Neural Network Control of Robot Arms. CIRP Annals 41(1): 407-410.

Sun Z and Roos N (2014) An energy efficient dynamic gait for a Nao robot. 2014 IEEE International Conference on Autonomous Robot Systems and Competitions, ICARSC 2014 267-272.

Sun Z and Roos N (2018) Dynamically stable walk control of biped humanoid on uneven and inclined terrain. Neurocomputing 280: 111-122.

Webots - Aldebaran 2.1.4.13 documentation. (n.d.). Retrieved February 14, 2020, from http://doc.aldebaran.com/21/software/webots/webots_index.html

Weerakoon T, Ishii K and Nassiraei AAF (2015) An Artificial Potential Field Based Mobile Robot Navigation Method To Prevent From Deadlock. Journal of Artificial Intelligence and Soft Computing Research 5(3): 189203. 